\title{
Contribution à la modélisation expérimentale et numérique des instabilités plastiques en hydroformage des tôles minces
}

\author{
Mahfoudh Ayadi $^{1}$, Mohamed Ali Rezgui ${ }^{1, a}$, Abel Cherouat ${ }^{2}$, Faouzi Slimani ${ }^{1}$ \\ ET Mohamed TOumi NASRI ${ }^{1}$ \\ 1 UR-MSSDT(99-UR11-46) b-ESSTT ${ }^{\mathrm{b}}$, 5 Av. Taha Hussein, Montfleury 1008, Tunis, Tunisie \\ 2 UTT-ICD/Projet GAMMA3 INRIA, Domaine de Voluceau-Rocquencourt, BP 105, 78153 Le Chesnay, France
}

Reçu le 21 mars 2009, accepté le 9 décembre 2009

\begin{abstract}
Résumé - Dans cette étude, on présente une contribution à la modélisation expérimentale et numérique du comportement élastoplastique endommageable anisotrope de tôles destinées à la mise en forme. L'accent est mis sur la localisation des instabilités plastiques induites par l'anisotropie plastique et l'endommagement. La procédure d'identification des paramètres matériau du modèle de comportement est basée sur une approche inverse d'optimisation s'appuyant sur des essais de traction orientés, associée à des simulations numériques (Abaqus/Standard et Matlab). La validation de la procédure proposée a pu être menée grâce à une confrontation entre simulations numériques et résultats expérimentaux obtenus sur des éprouvettes caractéristiques des tôles minces texturées en acier doux. L'application est ensuite faite à la simulation de procédés de gonflement hydraulique de ces tôles avec prévision de l'endommagement ductile. Plusieurs cas en expansion libre (matrices circulaires et elliptiques) et en expansion dans des cavités de matrice seront présentés et des comparaisons avec les résultats expérimentaux seront réalisées.
\end{abstract}

Mots clés : Hydroformage / écrouissage / anisotropie / endommagement / instabilité plastique / essais expérimentaux / éléments-finis

\begin{abstract}
Contribution of numerical and experimental modelling of plastic instabilities of thin sheets hydroforming. In this study, one presents experimental and numerical methodologies which aim to improve the plastic instabilities localisation induced during the $3 \mathrm{D}$ thin sheet hydroforming. This methodology is based on elastoplastic constitutive equations accounting for non-linear anisotropic hardening coupled to ductile damage. The experimental study is dedicated to the identification of material parameters from the global measure of tensile tests of anisotropic specimens. The validation of the procedure suggested could be carried out thanks to a confrontation between numerical simulations and experimental results obtained on thin sheets textured of mild steel. The application is then made to the simulation of hydroforming processes of these sheets with forecast of the ductile damage. Several cases of experimental and numerical tests free expansion with circular and elliptic dies and conditioned expansion in various dies are presented. Comparisons with the experimental results will be realized.
\end{abstract}

Key words: Hydroforming / hardening / anisotropy / damage / plastic instability / experimental tests / FEM

\footnotetext{
a Auteur pour correspondance :

mohamedali.rezgui@esstt.rnu.tn

b UR-MSSDT (99-UR11-46) : Unité de Recherche

Mécanique des Solides, des Structures et Développement

Technologique

c ESSTT : École Supérieure des Sciences et Techniques de Tunis
}

\section{Introduction}

De nos jours les besoins industriels dans des domaines très diversifiés tels que l'aéronautique, le nucléaire et l'automobile imposent des contraintes et des performances de plus en plus élevées afin de diminuer les coûts de fabrication et d'augmenter la fiabilité des pièces. La maîtrise de la prévision du comportement et de la rupture des structures mécaniques est devenue primordiale. Pour cette raison la simulation numérique occupe une place de plus 


\section{Nomenclature}

\begin{tabular}{|ll|}
\hline$a$ & Rapport force thermodynamique/déformation plastique cumulée \\
$D$ & Scalaire de dommage ductile isotrope \\
$F_{\text {exp }}^{i}$ & Tension expérimentale correspondant à $\delta_{i}[\mathrm{~N}]$ \\
$F_{\mathrm{num}}^{i}$ & Tension numérique correspondant à $\delta_{i}[\mathrm{~N}]$ \\
$\left(K, \varepsilon_{0}, n\right)$ & Coefficients d'écrouissage \\
$m$ & Nombre de mesures expérimentales \\
$F, G, H, N$ & Paramètres d'anisotropie \\
$Y$ & Force thermodynamique associée à l'endommagement \\
$f_{\mathrm{p}}$ & Partie plastique du pseudo-potentiel \\
$f_{\mathrm{d}}$ & Partie endommagement du pseudo-potentiel \\
$\sigma_{y}$ & Limite élastique du matériau [MPa] \\
$\tilde{\sigma}_{\mathrm{ij}}$ & Composantes du tenseur des contraintes effectives [MPa] \\
$\left(Y_{0}, \alpha, \beta\right)$ & Coefficients qui caractérisent la force thermodynamique associée à l'endommagement \\
$\mathrm{d} \lambda$ & Multiplicateur plastique \\
$\tilde{\sigma}_{\mathrm{eq}}$ & Contrainte équivalente effective associée à la surface de charge de Hill [MPa] \\
$\mathrm{d} \varepsilon_{\mathrm{eq}}^{\mathrm{p}}$ & Déformation plastique équivalente \\
$\mathrm{d} \varepsilon_{\mathrm{ij}}^{\mathrm{p}}$ & Composantes du tenseur de déformation plastique \\
$\left(\gamma, \varepsilon_{\mathrm{u}}, \varepsilon_{\mathrm{s}}\right)$ & Paramètres d'endommagement ductile \\
$\sigma_{\mathrm{eq}}$ & Contrainte équivalente de Von Mises [MPa] \\
$\delta i$ & Déplacement [mm] \\
$\xi_{\mathrm{F}}$ & Fonction objectif \\
\hline
\end{tabular}

en plus prépondérante dans certains domaines de l'industrie $[1,2]$. On assiste actuellement à un réel développement et à une utilisation systématique de méthodes numériques à chaque étape de la réalisation d'un produit (conception, fabrication, validation, essais, etc.) afin d'en optimiser le procédé de fabrication. Il est bien évident que l'utilisation du formage virtuel minimise les coûts engendrés par le choix des presses à utiliser, en calculant les efforts nécessaires durant la gamme de réalisation du produit, par le choix du processus de fabrication approprié et par l'optimisation des géométries des matrices...

Dans les procédés d'hydroformage des structures minces (tubes ou tôles), des études continuent à se développer pour cerner les principales causes de rupture et de défaillances des flans durant le processus de formage. Ces défaillances résultent, en grande partie, des instabilités plastiques associées à la présence de micro-défauts locaux qui engendrent l'incubation ou la nucléation, la coalescence et la croissance de l'endommagement. Pour la plupart des matériaux de mise en forme, la nucléation correspond à la décohésion de la matrice autour de certaines inclusions, de telles discontinuités conduisent à la création de microcavités ou de micro vides. Les effets cumulés des déformations et des tensions de la matrice provoquent une évolution des tailles et des formes de ces microcavités. Le stade de coalescence correspond à la phase de formation et de ramification des microfissures entre les micros vides. Enfin, la croissance résulte de la formation et la propagation d'une macro fissure prépondérante qui mène à la rupture finale du flan [3-9].

Les modèles prédictifs de l'endommagement sont des outils d'analyse exigés aussi bien par les ingénieurs que les industriels durant les différentes étapes de conception d'un nouveau produit. Une majorité de ces modèles est fondée sur une approche macroscopique dont la formulation est effectuée dans le cadre de la thermodynamique des processus irréversibles $[4,5,10,11]$ à travers l'introduction de variables d'état associées aux phénomènes mis en jeu.

Dans cette approche, on distingue deux démarches de modélisation. La première démarche qui est physique, est basée sur la notion du taux de croissance des cavités à l'intérieur d'une matrice à comportement élastoplastique $[12,13]$. L'endommagement est représenté par un scalaire utilisant la notion de fraction volumique de cavité, son évolution est liée à la croissance de ces défauts; elle est, par la suite, décomposée en trois phases allant de la formation d'une cavité jusqu'à l'amorçage d'une ou plusieurs fissures en passant par la phase de coalescence $[11,12]$. La seconde démarche définit la variable endommagement en tant qu'une diminution des sections actives $[7,8,13]$. Cette définition du dommage a permis d'introduire le concept de contraintes effectives à travers deux principes d'équivalence dont le premier est exprimé en terme de déformations $[11,12]$ et le second est exprimé en terme d'énergie $[13,14]$.

Les différents concepts utilisés pour décrire l'endommagement sont à l'origine des études orientées vers le développement des procédés de formage des tubes et des tôles. Dans ces études, les formulations analytiques des lois de comportement sont exploitées pour construire des modèles numériques capables de simuler le processus de formage et de prédire et localiser les éventuelles zones de rupture [15-18, 20-23].

Ce travail est dédié à l'étude d'une approche « avancée » basée sur une modélisation pour la simulation 
numérique des procédés de mise en forme par hydroformage des tôles et leur optimisation vis-à-vis de l'endommagement ductile. En particulier nous nous intéressons à l'étude des effets de l'expansion des flans avec ou sans matrice et du couplage comportement endommagement en grandes déformations sur la faisabilité du procédé de mise en forme.

\section{2 Étude théorique}

Le comportement élastoplastique couplé à l'endommagement est décrit dans le cadre de la thermodynamique des processus irréversibles où la dissipation mécanique est décrite dans le cadre d'une théorie à une surface qui considère un seul mécanisme pour représenter à la fois la plasticité et l'endommagement $[2,3,7]$. Ceci permet de décomposer le pseudo potentiel en une partie plastique $f_{\mathrm{p}}$ et une partie endommagement $f_{\mathrm{d}}$. Le critère de Hill [19] couplé à un endommagement ductile isotrope représenté par le scalaire $D$, est utilisé pour définir la surface de charge $f_{\mathrm{p}}$ qui, en contraintes planes (tôles minces), admet pour expression :

$$
\begin{aligned}
& f_{\mathrm{p}}\left(\tilde{\sigma}_{i j}, \sigma_{y}\right)= \\
& \sqrt{F \tilde{\sigma}_{22}^{2}+G \tilde{\sigma}_{11}^{2}+H\left(\tilde{\sigma}_{22}-\tilde{\sigma}_{11}\right)^{2}+2 N \tilde{\sigma}_{12}^{2}}-\sigma_{y}=0
\end{aligned}
$$

où $\sigma_{y}$ est la limite élastique du matériau, $(F, G, H, N)$ sont les paramètres d'anisotropie et $\tilde{\sigma}_{i j}$ sont les composantes du tenseur des contraintes effectives de Cauchy, elles sont reliées aux contraintes apparentes $\sigma_{i j}$ par la relation :

$$
\tilde{\sigma}_{i j}=\frac{\sigma_{i j}}{1-D}
$$

Pour représenter la partie endommagement $f_{\mathrm{d}}$ plusieurs modèles sont utilisés dans la littérature $[2,9,10]$. Dans cette étude, nous considérons que l'évolution de l'endommagement est liée à la valeur de sa variable associée $Y$ représentant le taux de restitution d'énergie mécanique dans le matériau, et également fonction de l'accroissement de la déformation plastique :

$$
f_{\mathrm{d}}(D, Y)=\frac{\left(Y+Y_{0}\right)^{\alpha}}{D^{\beta}}
$$

où $Y$ est la force thermodynamique associée à l'endommagement $D,\left(Y_{0}, \alpha, \beta\right)$ sont des coefficients qui caractérisent l'endommagement ductile du matériau.

La règle de normalité permet d'établir les relations complémentaires suivantes :

$$
\begin{aligned}
\mathrm{d} \varepsilon_{i j}^{\mathrm{p}} & =\frac{\partial f_{\mathrm{p}}}{\partial \sigma_{i j}} \mathrm{~d} \lambda \\
\mathrm{d} D & =\frac{\partial f_{\mathrm{d}}}{\partial Y} \mathrm{~d} \lambda
\end{aligned}
$$

où $\mathrm{d} \lambda$ représente le multiplicateur plastique, donné par la condition d'équivalence de l'énergie plastique :

$$
\tilde{\sigma}_{\text {eq }} \mathrm{d} \varepsilon_{\text {eq }}^{\mathrm{p}}=\tilde{\sigma}_{i j} \mathrm{~d} \varepsilon_{i j}^{\mathrm{p}}
$$

La contrainte équivalente effective et la déformation plastique équivalente associées à la surface de charge de Hill sont définies dans le cas des tôles minces par [19] :

$$
\tilde{\sigma}_{\mathrm{eq}}=\sqrt{F \tilde{\sigma}_{22}^{2}+G \tilde{\sigma}_{11}^{2}+H\left(\tilde{\sigma}_{22}-\tilde{\sigma}_{11}\right)^{2}+2 N \tilde{\sigma}_{12}^{2}}
$$

ce qui conduit à l'expression du multiplicateur plastique $\mathrm{d} \lambda$ :

$$
\mathrm{d} \lambda=\mathrm{d} \varepsilon_{\mathrm{eq}}^{\mathrm{p}}
$$

Compte tenu des équations (3) et (8), l'évolution de l'endommagement $D$ est fonction de la force thermodynamique $Y$ et de l'accroissement de la déformation plastique cumulée :

$$
\mathrm{d} D=\frac{\alpha\left(Y+Y_{0}\right)^{\alpha-1}}{D^{\beta}} \mathrm{d} \lambda
$$

Dans le cadre de cette étude, nous proposons la force thermodynamique associée à la variable d'endommagement comme une fonction linéaire d'une variable observable telle que la déformation plastique équivalente cumulée :

$$
Y=a \varepsilon_{\mathrm{eq}}^{\mathrm{p}}
$$

Alors la relation (9) devient intégrable et le dommage $D$ pourrait être mesuré à partir de $\varepsilon_{\mathrm{eq}}^{\mathrm{p}}$, en considérant la relation :

$$
D=\left[\frac{\beta+1}{a}\left(a \varepsilon_{\mathrm{eq}}^{\mathrm{p}}+Y_{0}\right)^{\alpha}\right]^{1 / \beta+1}
$$

Il en résulte que l'état vierge du matériau qui correspond à une déformation plastique équivalente nulle, est caractérisé par un dommage $D_{0}$ lié aux micro-défauts initiaux du matériau et donné par la relation :

$$
D_{0}=\left[\frac{\beta+1}{a} Y_{0}^{\alpha}\right]^{1 / \beta+1}
$$

Finalement l'expression de l'endommagement ductile isotrope s'écrit sous la forme simplifiée suivante :

$$
D=\left[\frac{\varepsilon_{\mathrm{eq}}^{\mathrm{p}}+\varepsilon_{\mathrm{s}}}{\varepsilon_{\mathrm{u}}}\right]^{\gamma}
$$

où les paramètres $\left(\varepsilon_{\mathrm{u}}, \varepsilon_{\mathrm{s}}\right)$ sont équivalents à des déformations, ils caractérisent avec $\gamma$ l'évolution de l'endommagement dans le matériau. Ils sont reliés entre eux par les relations :

$$
\left\{\begin{array}{l}
\varepsilon_{\mathrm{u}}=(1+\beta)^{-1 / \alpha} a^{(1-\alpha) / \alpha} \\
\frac{\varepsilon_{\mathrm{s}}}{\varepsilon_{\mathrm{u}}}=D_{0}^{1 / \gamma} \\
\gamma=\alpha /(1+\beta)
\end{array}\right.
$$

Finalement, trois groupes d'inconnues doivent être déterminés, pour connaître le comportement global du matériau :

1. Quatre paramètres d'anisotropie : $F, G, H$ et $N$.

2. Trois paramètres d'endommagement ductile isotrope : $\gamma, \varepsilon_{\mathrm{s}}, \varepsilon_{\mathrm{u}}$. 
Tableau 1. Résultats de l'identification du modèle de comportement.

\begin{tabular}{ccc}
\hline \multirow{2}{*}{ Caractéristiques élastiques } & Module d'élasticité [GPa] & 210 \\
& Coefficient de Poisson & 0,33 \\
Limite élastique & 195 \\
\hline \multirow{2}{*}{ Coefficients de la loi d'écrouissage non } & $\mathrm{K}(\mathrm{MPa})$ & 497,8 \\
couplée & $\varepsilon_{0}$ & 0,012 \\
& $n$ & 0,2187 \\
\hline \multirow{2}{*}{ Paramètres d'anisotropie } & $F$ & 0,665 \\
& $G$ & 0,335 \\
& $H$ & 0,406 \\
& $N$ & 1,285 \\
\hline \multirow{2}{*}{ Coefficients de Lankford } & $r_{0}$ & 1,985 \\
& $r_{45}$ & 1,234 \\
& $r_{90}$ & 1,637 \\
\hline
\end{tabular}

3. Trois paramètres d'écrouissage du modèle de Swift $\left(K, \varepsilon_{0}, n\right)$ reliant l'évolution de la limite élastique à la déformation plastique équivalente cumulée par la relation :

$$
\sigma_{y}=K\left(\varepsilon_{0}+\varepsilon_{\mathrm{eq}}^{\mathrm{p}}\right)^{n}
$$

La détermination des dix paramètres matériau est fondée sur des mesures expérimentales des grandeurs apparentes. Dans le cas du couplage effectif entre élastoplasticité et endommagement, la contrainte équivalente est reliée à la limite élastique par la formule :

$$
\sigma_{\mathrm{eq}}=(1-D) \sigma_{y}
$$

La loi d'écrouissage couplée à l'endommagement pourra se présenter sous la forme:

$$
\sigma_{\mathrm{eq}}=K\left[1-\left(\frac{\varepsilon_{\mathrm{eq}}^{\mathrm{p}}+\varepsilon_{\mathrm{s}}}{\varepsilon_{\mathrm{u}}}\right)^{\gamma}\right]\left(\varepsilon_{0}+\varepsilon_{\mathrm{eq}}^{\mathrm{p}}\right)^{n}
$$

\section{Démarche d'identification}

Dans ce qui suit, nous décrivons la procédure utilisée pour identifier l'ensemble des paramètres du modèle de comportement élastoplastique anisotrope couplé à un endommagement ductile isotrope d'une tôle en acier doux type E24. Les paramètres d'anisotropie ainsi que les coefficients d'écrouissage de la tôle sont déterminés à partir des courbes rationnelles des essais de traction orientée, alors que les courbes forces/allongement des mêmes essais sont utilisées pour construire, moyennant des simulations numériques, le modèle d'écrouissage couplé qui permet de décrire le meilleur du comportement du matériau $[22,24,25]$.

\subsection{Paramètres d'anisotropie et coefficients d'écrouissage}

Les essais de traction effectués sur des éprouvettes normalisées classiques (épaisseur $e=1 \mathrm{~mm}$, hauteur $h=$ $12,52 \mathrm{~mm}$ et longueur initiale $l_{0}=80 \mathrm{~mm}$ ) et orientées à $0^{\circ}, 45^{\circ}$ et $90^{\circ}$ par rapport à la direction de laminage de la tôle, ont été exploités pour caractériser l'anisotropie et l'écrouissage de la tôle. En considérant la direction de laminage comme référence $(G+H=1)$, les coefficients $K, n$ et $\varepsilon_{0}$ ont pu être déterminés à partir de la courbe rationnelle de traction à $0^{\circ}$. La meilleure corrélation, entre expérience et modèle de Swift non couplé représenté par la relation (15), a été cherchée dans une limite de déformation plastique inférieure à $15 \%$, dans cet intervalle l'effet du dommage est ignoré.

Les paramètres de la surface de charge de Hill représentant l'anisotropie de la tôle sont déterminés en considérant les coefficients de Lankford $\left(r_{0}, r_{90}, r_{45}\right)$ évalués expérimentalement, avec :

$$
\left\{\begin{array}{l}
F=\frac{r_{0}}{r_{90}\left(1+r_{0}\right)} \\
G=1 /\left(1+r_{0}\right) \\
H=r_{0} /\left(1+r_{0}\right) \\
N=\frac{\left(r_{0}+r_{90}\right)\left(2 r_{45}+1\right)}{2 r_{90}\left(1+r_{0}\right)}
\end{array}\right.
$$

Le tableau 1 récapitule les différentes caractéristiques obtenues après cette première étape d'identification, les courbes de contrainte équivalente/déformation plastique équivalente cumulée (Fig. 1) permettent de comparer les prévisions du modèle d'écrouissage identifié aux résultats expérimentaux pour deux directions de laminage $D L=$ $0^{\circ}$ et $D L=45^{\circ}$. Notons que les résultats de l'identification sont les valeurs moyennes des coefficients qui régissent le modèle suivant les directions de l'anisotropie. L'expérience montre que la rupture survient à $32 \%$ et la contrainte maximale atteinte est de l'ordre de $488 \mathrm{MPa}$ pour une direction du laminage $D L=45^{\circ}$ et de l'ordre de $358 \mathrm{MPa}$ pour $D L=0^{\circ}$. Le calcul d'identification reproduit avec fidélité le comportement réel du matériau jusqu'à une déformation de $27 \%$. Au-delà de cette limite, on constate une légère différence des résultats.

\subsection{Paramètres d'endommagement}

Deux conditions ont été imposées, pour limiter les domaines de recherche des coefficients d'endommagement $\left(\gamma, \varepsilon_{\mathrm{s}}, \varepsilon_{\mathrm{u}}\right):$

1. La première est introduite par un critère d'instabilité plastique, celui que nous considérons est 
Tableau 2. Déformations et contraintes critiques expérimentales.

\begin{tabular}{cccc}
\hline Angles par rapport à la direction de laminage de la tôle & $0^{\circ}$ & $45^{\circ}$ & $90^{\circ}$ \\
\hline Déformation critique expérimentale [\%] & 27,2 & 31,0 & 25,6 \\
Contrainte critique expérimentale [MPa] & 374 & 365 & 363 \\
\hline
\end{tabular}

Tableau 3. Effet des coefficients du modèle d'endommagement.

\begin{tabular}{cccccc}
\hline Modèle & $\varepsilon_{\mathrm{s}}[\%]$ & $\varepsilon_{\mathrm{u}}[\%]$ & $\gamma$ & Déformation critique [\%] & Contrainte critique [MPa] \\
\hline 1 & 5,0 & 50 & 7,0 & 25,8 & 362,0 \\
2 & 5,5 & 55 & 8,0 & 30,2 & 375,2 \\
3 & 6,0 & 65 & 9,0 & 37,7 & 394,5 \\
4 & 6,3 & 60 & 10,0 & 35,3 & 390,0 \\
5 & 6,5 & 62 & 10,6 & 37,3 & 394,0 \\
\hline
\end{tabular}

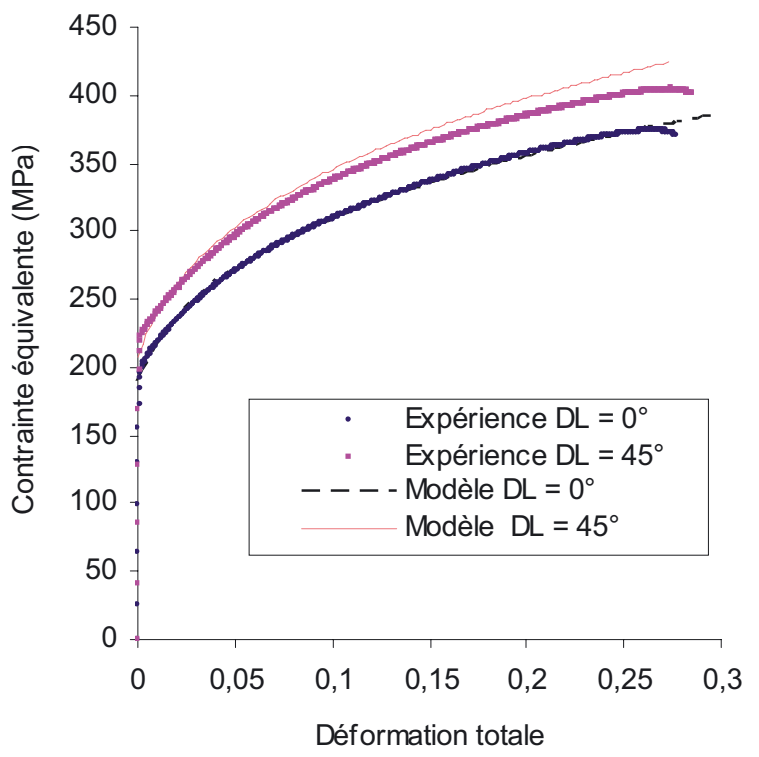

Fig. 1. Identification du modèle d'écrouissage avec $D L=0^{\circ}$ et $D L=45^{\circ}$.

défini par l'extremum du modèle d'écrouissage couplé (Éq. (17)) :

$$
\frac{\mathrm{d} \sigma_{\mathrm{eq}}}{\mathrm{d} \varepsilon_{\mathrm{eq}}^{\mathrm{p}}}=0
$$

La condition d'extremum (19), montre que l'instabilité plastique survient au moment où la déformation plastique cumulée atteint une valeur critique $\left(\varepsilon_{\mathrm{c}}^{\mathrm{p}}\right)$ vérifiant l'équation :

$$
\left(\frac{\varepsilon_{\mathrm{s}}+\varepsilon_{\mathrm{c}}^{\mathrm{p}}}{\varepsilon_{\mathrm{u}}}\right)^{\gamma-1}\left(n+\gamma \frac{\varepsilon_{0}+\varepsilon_{\mathrm{c}}^{\mathrm{p}}}{\varepsilon_{\mathrm{u}}}\right)-n=0
$$

2. La seconde est imposée par les valeurs de la variable de dommage $D$ qui devront être comprises entre 0 (matériau sain) et 1 (matériau totalement endommagé). Ceci n'est possible que si l'inéquation (21) est satisfaite :

$$
\varepsilon_{\mathrm{s}}+\varepsilon_{\mathrm{eq}}^{\mathrm{p}}<\varepsilon_{\mathrm{u}}
$$

Le coefficient $\varepsilon_{\mathrm{u}}$ pourra ainsi représenter la déformation plastique totale cumulée à la rupture majorée de $\varepsilon_{\mathrm{s}}$ et minorée par $\varepsilon_{\mathrm{s}}+\varepsilon_{\mathrm{c}}^{\mathrm{p}}$. Par ailleurs, comme les déformations limites en expansion biaxiale dépassent largement celles de la traction simple, cela nous autorise à choisir la valeur minimale de la déformation $\varepsilon_{\mathrm{c}}^{\mathrm{p}}$ en tant que majorant des déformations critiques déduites expérimentalement des essais de traction orientée. En effet, compte tenu des paramètres d'anisotropie (Tab. 1), les contraintes et les déformations équivalentes critiques ont pu être calculées. Les résultats obtenus pour les trois directions du laminage sont présentés dans le tableau 2 .

La condition imposée à $\varepsilon_{\mathrm{c}}^{\mathrm{p}}$, permet en effet de réduire la taille du domaine de recherche des coefficients du dommage. Ainsi, seules les combinaisons entre $\gamma, \varepsilon_{\mathrm{s}}$ et $\varepsilon_{\mathrm{u}}$ de l'équation (20) conduisant à des solutions en $\varepsilon_{\mathrm{c}}^{\mathrm{p}}$ supérieures à $31 \%$ seront considérées dans la suite de l'identification.

Le problème d'identification consiste à trouver la meilleure combinaison des paramètres endommagement $\left(\gamma, \varepsilon_{\mathrm{s}}\right.$ et $\left.\varepsilon_{\mathrm{u}}\right)$ qui minimise l'écart $\left(\xi_{\mathrm{F}}\right)$ entre prévisions numériques et résultats expérimentaux $[22,26]$. Cette minimisation a concerné les écarts entre les $m$ mesures expérimentales des tensions fonction de l'allongement et leurs prévisions numériques effectuées sur des éprouvettes de traction. La fonction objectif utilisée à cet effet est définie par :

$$
\xi_{\mathrm{F}}=\frac{1}{m} \sqrt{\sum_{i=1}^{m}\left(\frac{F_{\mathrm{exp}}^{\mathrm{i}}-F_{\mathrm{num}}^{\mathrm{i}}}{F_{\mathrm{exp}}^{\mathrm{i}}}\right)^{2}}
$$

où $F_{\exp }^{\mathrm{i}}$ et $F_{\text {num }}^{\mathrm{i}}$ sont respectivement la tension expérimentale et numérique correspondant au même allongement $\delta_{\mathrm{i}}$.

Les simulations numériques des essais de traction uniaxiale orientée sont effectuées sur des éprouvettes normalisées encastrées en une extrémité et soumises à un déplacement longitudinal de $30 \mathrm{~mm}$. Les calculs $3 \mathrm{D}$ en grandes déformations sont réalisés sur $\mathrm{ABA}$ QUS/Standard [23]. Les éprouvettes sont discrétisées avec 3108 éléments tétraédriques (C3D4) de la bibliothèque des éléments. Des scripts python sont utilisés en utilisant ABAQUS/Standard et Matlab pour optimiser (méthode du simplex) les paramètres matériau sur les réponses globales tension-allongement $[25,27,28]$. Cinq jeux de paramètres d'endommagement sont proposés pour illustrer 


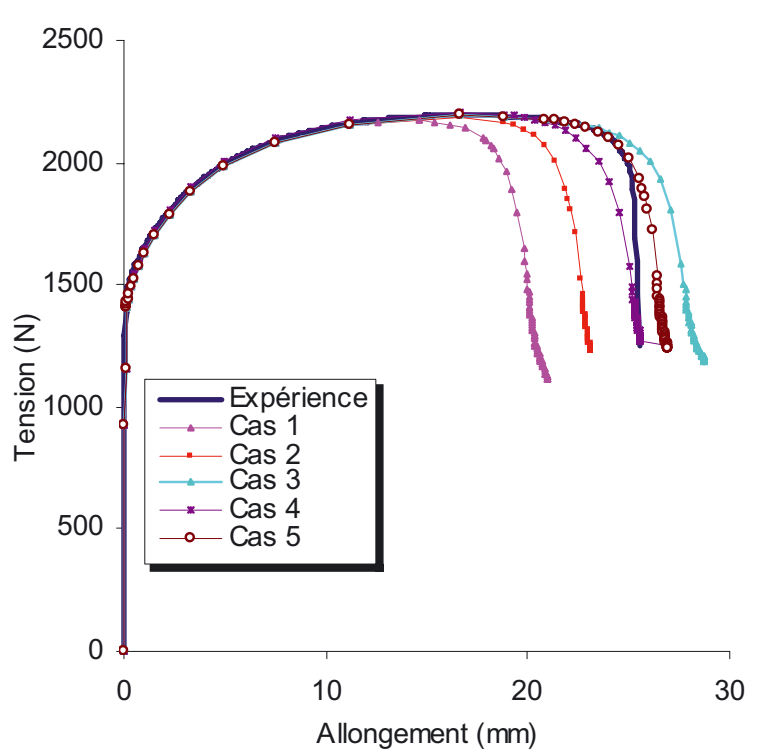

(a) $\mathrm{DL}=0^{\circ}$

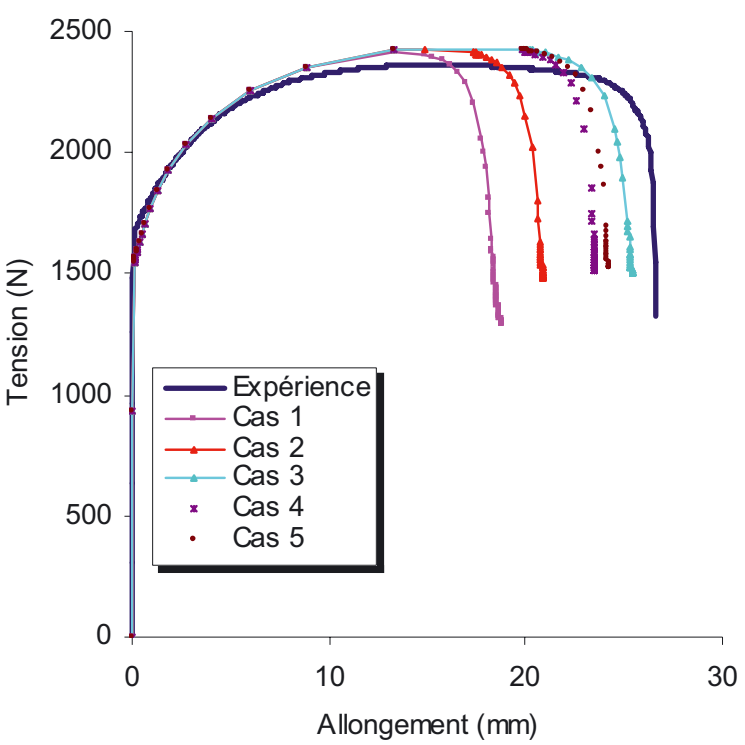

(b) $\mathrm{DL}=45^{\circ}$

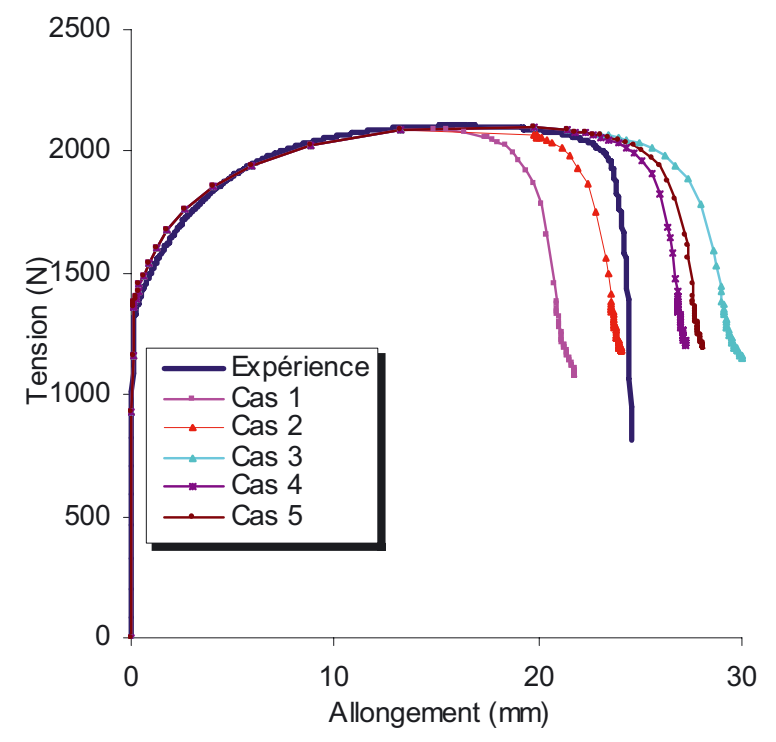

(c) $\mathrm{DL}=90^{\circ}$

Fig. 2. Courbes tension/allongement pour différents modèles d'écrouissage.

leur effet du dommage sur la réponse locale du matériau (voir Tab. 3).

La figure 2 illustre des exemples de simulations réalisées avec cinq jeux de paramètres d'écrouissage donnés dans le tableau 1 (pour l'élasticité et la plasticité) et tableau 3 (pour l'endommagement).

Les résultats obtenus montrent que les coefficients d'endommagement du modèle type 5, associé à la loi d'écrouissage de Swift, présentent aussi bien en terme de contrainte/déformation qu'en terme de tension/allongement, le meilleur compromis avec les résultats expérimentaux. En considérant la direction de laminage comme référence, l'essai de traction à $0^{\circ}$ a pu servir à la fois pour identifier les coefficients de la loi de Swift non couplé et pour le calage du modèle avec endommagement, c'est ce qui justifie les écarts relativement faibles enregistrés entre expérience et simulation. Le tableau 4 regroupe à cet effet les écarts relatifs des prévisions numériques par rapport aux résultats d'essai. La comparaison concerne particulièrement les tensions maximales et les allongements à la rupture. Il y a lieu de constater que les écarts relatifs les plus faibles sont enregistrés entre les tensions maximales qui évoluent entre 0,14 et $3,2 \%$ avec un désavantage du côté de la traction à $45^{\circ}$, alors que les écarts relatifs les plus élevés correspondent aux allongements à la rupture qui se situent entre 5,24 et $14,7 \%$ avec un désavantage du côté de la traction à $90^{\circ}$.

La figure 3 permet, par ailleurs, de visualiser pour différentes amplitudes d'allongement les isovaleurs de la déformation plastique cumulée ainsi que le début de la 


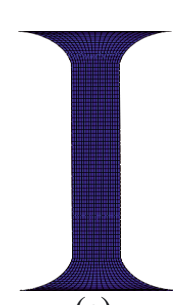

(a)

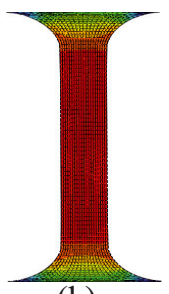

(b)

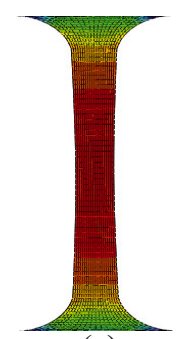

(c)

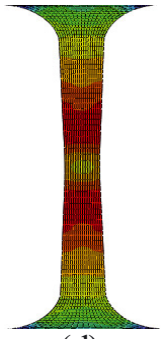

(d)

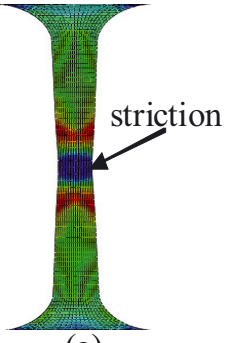

(e)

Fig. 3. Iso-valeurs de la déformation plastique cumulée à différentes amplitudes d'allongements, (a) initiale; (b) 5,1 mm; (c) $22,4 \mathrm{~mm}$; (d) $25,6 \mathrm{~mm}$; (e) $26,7 \mathrm{~mm}$.

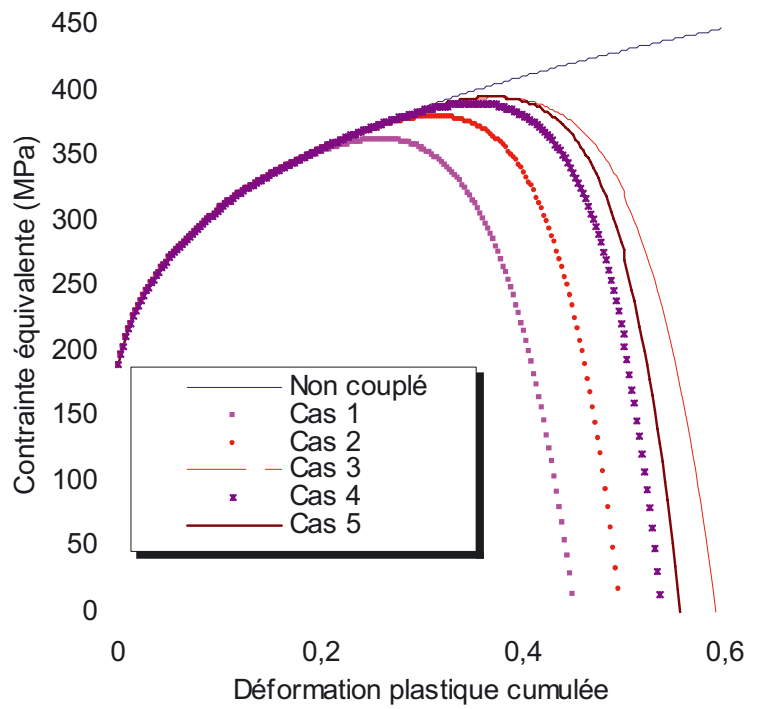

Fig. 4. Contrainte de Von Mises - déformation plastique cumulée calcul avec et sans couplage.

striction localisée. On note que ce calcul couplant la plasticité à l'endommagement reproduit fidèlement les différentes étapes de la rupture de l'éprouvette. En particulier l'état de déformation et d'endommagement commence par être très homogène dans la partie centrale de l'éprouvette (Fig. 3c) pour un déplacement $u=22,4 \mathrm{~mm}$. Ensuite pour un déplacement $u=25,6 \mathrm{~mm}$ on observe le début de la striction diffuse (Fig. 3d). La striction localisée apparaît clairement sur la figure 3e où l'on remarque la localisation dans la bande de cisaillement suivant la première bissectrice pour un déplacement $u=26,7 \mathrm{~mm}$. L'amorçage d'une fissure macroscopique apparaît à l'intersection de ces deux bandes de cisaillement et se propage dans les deux sens de la même bande jusqu'à la rupture finale de l'éprouvette.

L'évolution de la contrainte de Von Mises dans le cas d'un calcul avec ou sans couplage avec le dommage pour différentes valeurs de $\varepsilon_{\mathrm{u}}$ est illustrée sur la figure 4. Dans le cas du calcul non couplé l'endommagement n'est pas pris en compte et le matériau est supposé rester sain durant tout le chargement. Dans le cas 1 (peu écrouissable), le matériau présente une déformation plastique à la rupture $\varepsilon_{\mathrm{u}}=50 \%$ avec une contrainte maximale atteinte de l'ordre de $362 \mathrm{MPa}$ alors que dans le cas 3 (fortement

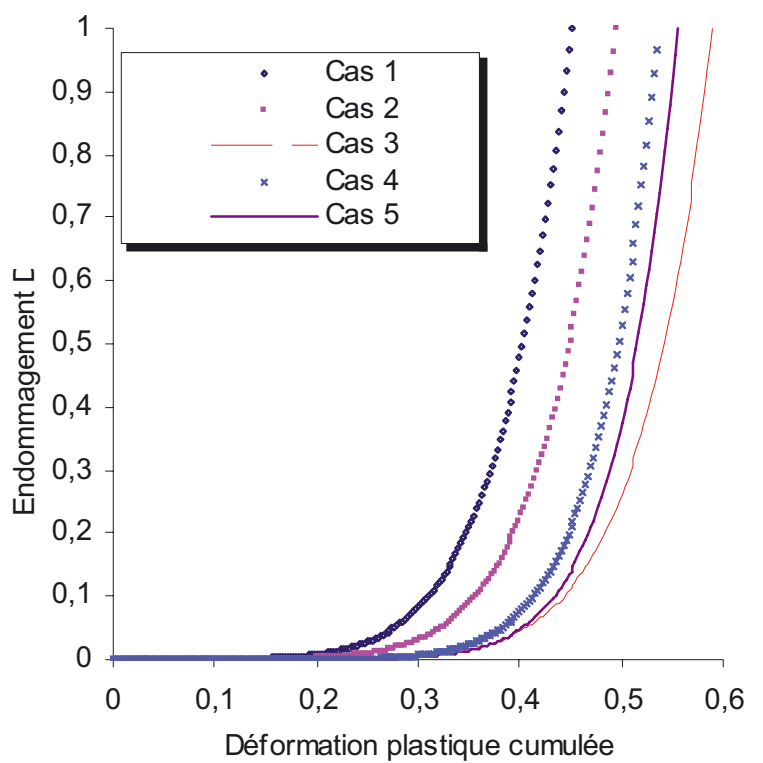

Fig. 5. Évolution de l'endommagement $D$ en fonction de la déformation plastique cumulée.

écrouissable), il présente une rupture à une déformation de $\varepsilon_{\mathrm{u}}=65 \%$ et une contrainte maximale de l'ordre de 394 MPa. L'évolution de l'endommagement en fonction de la déformation plastique cumulée pour différentes valeurs du coefficient de dommage $\gamma$ est illustrée sur la figure 5 . On note que plus le coefficient de dommage $\gamma$ augmente et plus la vitesse de croissance de l'endommagement est grande.

\section{Applications au procédé d'hydroformage}

Dans cette section nous présentons, en utilisant le modèle de comportement identifié précédemment, les résultats expérimentaux et les simulations numériques de l'hydroformage des tôles minces en expansion libre ou avec matrices (voir Fig. 6a) :

(i) Expansion libre avec deux formes de serre flan (voir Figs. $6 \mathrm{~b}$ et c) :

a. Serre-flan circulaire (CC) avec un évidement également circulaire de diamètre intérieur de $220 \mathrm{~mm}$ et un diamètre extérieur de $330 \mathrm{~mm}$. 


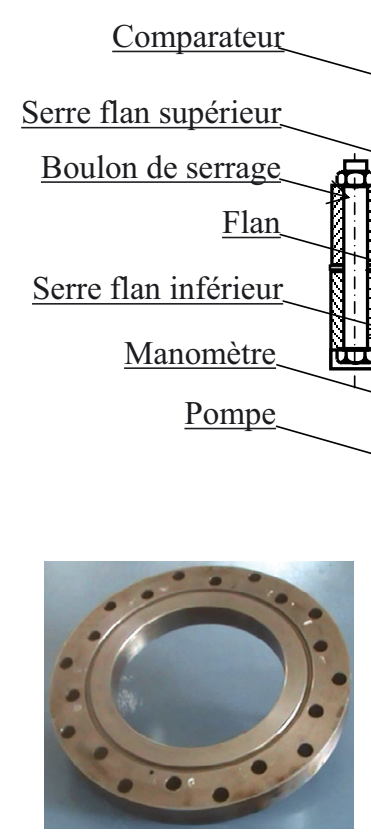

(b) Cavité circulaire $\mathrm{CC}$
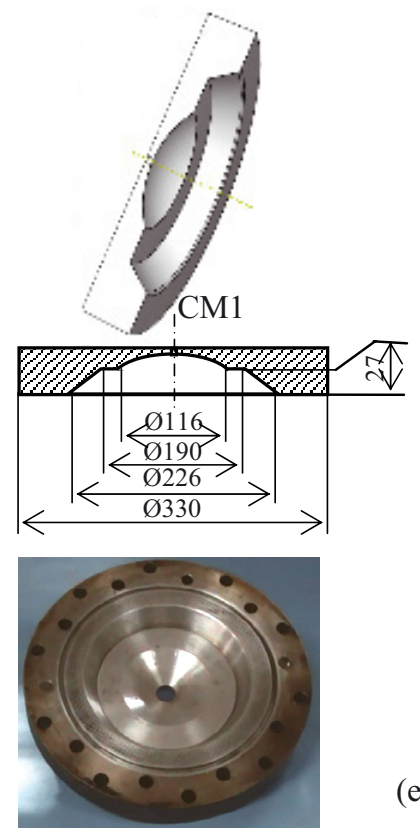

(d) Cavité de matrice CM1 (c) Cavité Elliptique CE
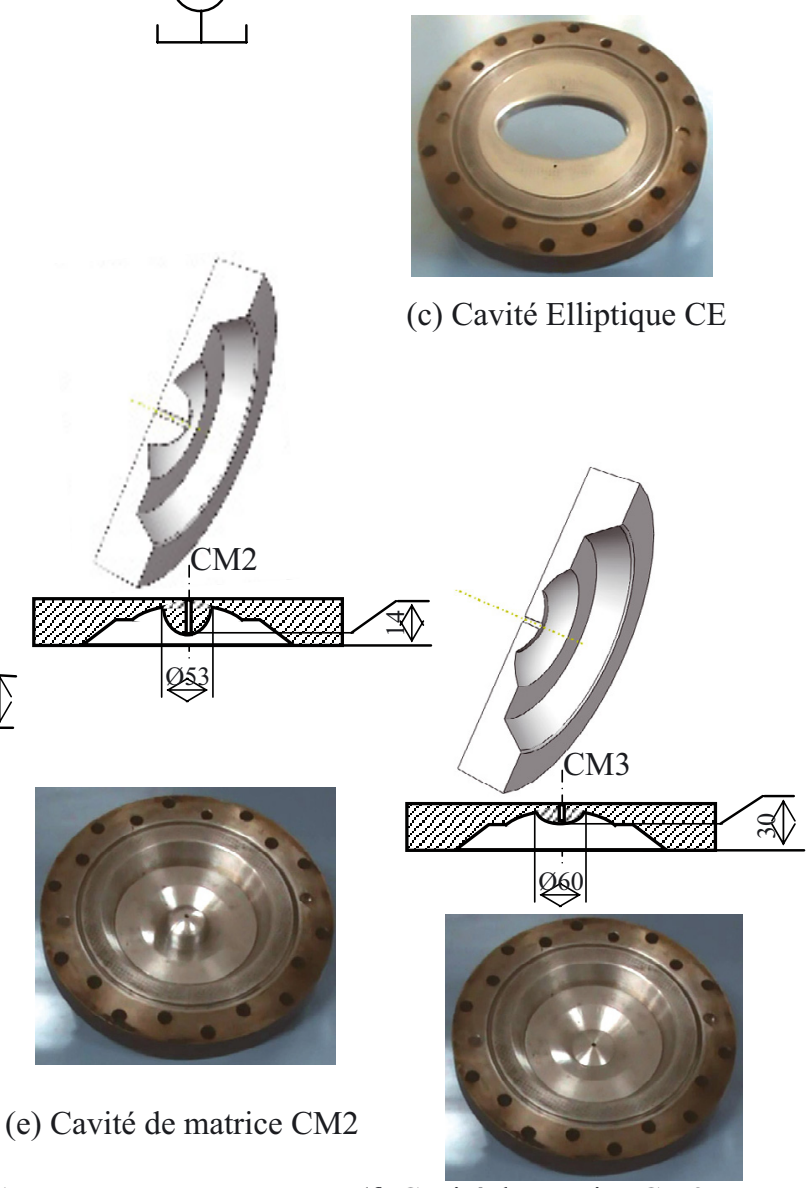

(a) Dispositif d'hydroformage

(f) Cavité de matrice CM3

Fig. 6. Dispositif d'hydroformage et géométries des cavités de matrices.

b. Serre-flan elliptique (CE) de même diamètre extérieur que le premier, mais il comporte un évidement elliptique (petit diamètre $=102 \mathrm{~mm}$ et grand diamètre $=170 \mathrm{~mm}$ ).

(ii) Expansion dans trois cavités de matrice (voir Fig. 6d pour matrice CM1, Fig. 6e pour matrice CM2 et Fig. 6f pour matrice CM3) formées d'une succession de surfaces de révolution (conique, plane, sphérique concave et convexe).

Tous les essais sont menés sur des plaques (flans) circulaires orthotropes de diamètre $300 \mathrm{~mm}$ et d'épaisseur
0,6 mm. La pression appliquée au flan est assurée par l'intermédiaire d'un liquide de transmission injecté avec un débit constant d'environ $10 \mathrm{ml} \cdot \mathrm{min}^{-1}$. Durant le processus, le déplacement au pôle en fonction de la pression a pu être enregistré expérimentalement.

Les simulations numériques des différentes applications sont effectuées sur Abaqus/Standard, en considérant le serrage du flan et son expansion au cours du temps. Le comportement de la tôle est élastoplastique anisotrope couplé à l'endommagement (modèle d'écrouissage cas 5). La tôle est maillée avec des éléments solides 3D 

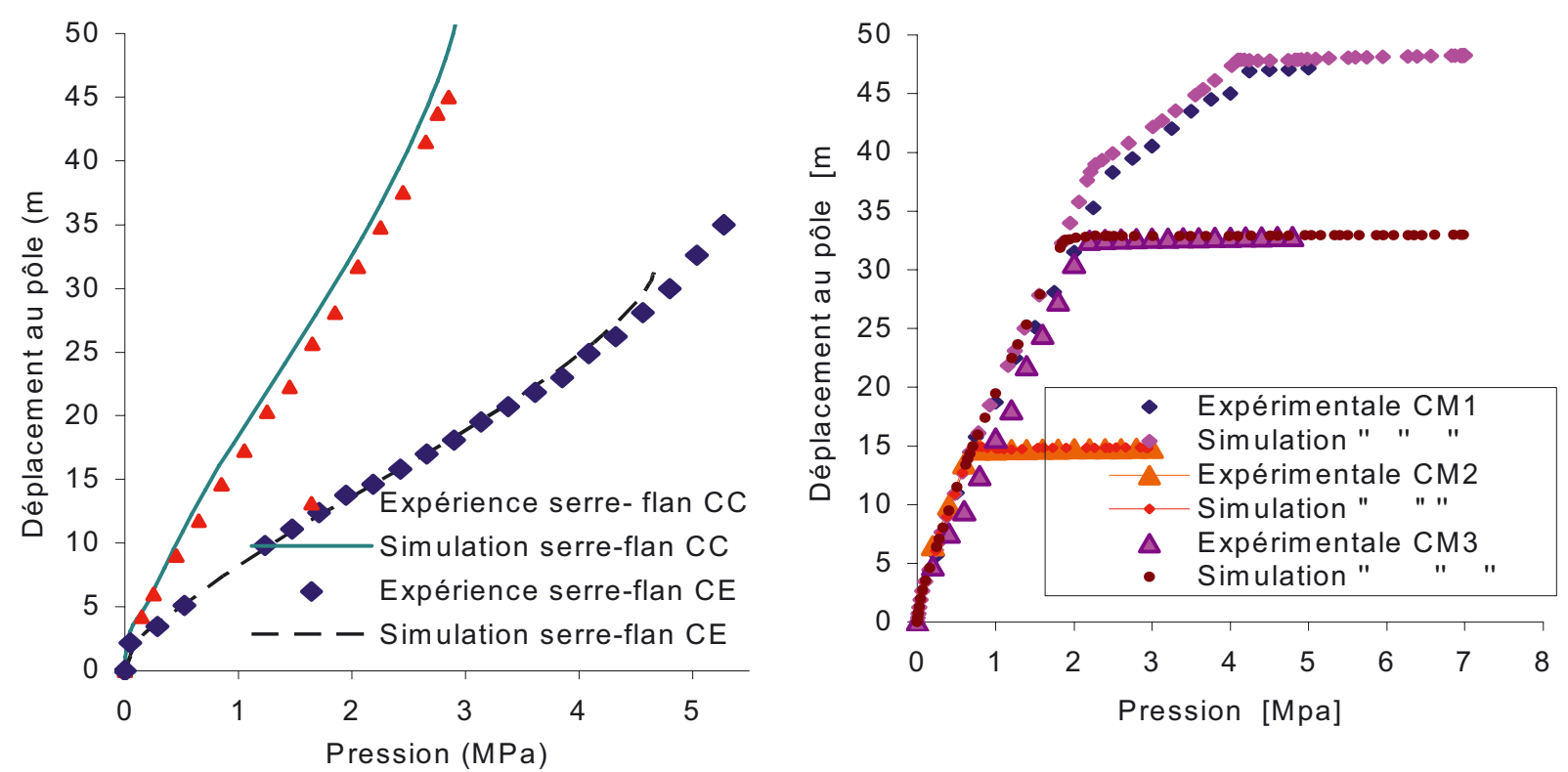

Fig. 7. Déplacements au pôle en fonction de la pression, (a) expansion libre, (b) expansion avec cavités de matrice.

linéaires hexaédriques à intégration réduite C3D8R et des éléments tétraédriques C3D6 de la librairie d'éléments d'ABAQUS. La tôle est rangée dans son épaisseur par deux couches d'éléments. Les matrices sont supposées rigides analytiques non discrétisées. Le nombre d'éléments est de l'ordre de 4144 pour l'expansion libre et de l'ordre de 5177 pour l'expansion dans les cavités de matrice.

\subsection{Confrontation résultats expérimentaux/prédictions numériques}

Les confrontations des résultats expérimentaux/prédictions numériques concernent deux séries d'informations.

\subsubsection{Résultats quantitatifs}

Dans cette partie nous nous sommes intéressés particulièrement à l'évolution du déplacement aux pôles en fonction de la pression durant l'essai de formage ainsi qu'aux profils de la tôle déformée et des épaisseurs à la fin de l'essai. Les profils des déplacements sont reconstitués moyennant un scanner 3D de type Dr. Picza Roland d'une précision de $5 \mu \mathrm{m}$ avec un pas d'effleurement réglé à $5 \mathrm{~mm}$. Par ailleurs, deux techniques de mesures ont été utilisées pour évaluer l'amincissement de la tôle après formage : (i) une technique non destructive utilisant une source ultrasonique de marque Sofranel (Model 26MG) ; (ii) une technique destructive utilisant un palmer digital de marque Mitiyuta de précision $10 \mu \mathrm{m}$ [26, 29]. La confrontation des deux techniques, sur la base de la répétitivité des résultats nous a conduits à privilégier la seconde technique. En effet, les mesures ultrasoniques de l'épaisseur semblent être fortement perturbées par les courbures de la surface palpée de la tôle et des défauts de positionnement de l'émetteur ultrasonique.

Les résultats présentés dans la figure 7 montrent que les écarts relatifs entre simulation numérique et expérience (expansion libre et dans les cavités de matrice) restent, pour les déplacements au pôle, dans la limite de $7 \%$ alors que les niveaux de pression sont en dessous d'un seuil caractérisant le type d'application.

La figure 8 illustre les profils de la tôle déformée (simulation et expérience par scannage) dans le cas d'un hydroformage en expansion libre avec serre flan type $\mathbf{C E}$ et d'un hydroformage en expansion avec cavité de matrice type CM3. On note que l'application relative à l'expansion libre CE (Fig. 8a) enregistre un décalage important entre simulation et mesure, localisé au niveau du pôle, cela peut être justifié par l'étirement que subit la tôle pendant la phase de l'éclatement brusque. En effet, le pôle est la région la plus exposée à un amincissement sévère conduisant à une chute significative de la raideur locale de la tôle. Cela engendre des déformations supplémentaires qui viennent modifier l'aspect final de la déformée. Cependant, le formage avec cavité de matrice CM3 (Fig. 8b) montre une cohérence entre simulation et mesure ce qui signifie que bien qu'il y ait eu striction et rupture, la marge des déplacements s'est trouvée réduite, la matrice a pu jouer le rôle de raidisseur de la tôle.

L'évolution des épaisseurs, dans le cas d'un hydroformage avec serre flan (CE) suivant le demi grand axe et le demi petit axe, est reportée sur la figure 9. Les valeurs des épaisseurs obtenues par simulation correspondent à une pression de 4,79 $\mathrm{MPa}$ (voir Fig. 7a) et celles mesurées sont obtenues après éclatement. Les résultats numériques montrent que l'épaisseur minimale au début de la striction est l'ordre de 0,42 $\mathrm{mm}$, alors que la mesure expérimentale montre que celle-ci atteint $0,38 \mathrm{~mm}$ à la rupture, ce qui représente un écart relatif d'environ 10,5\%. 

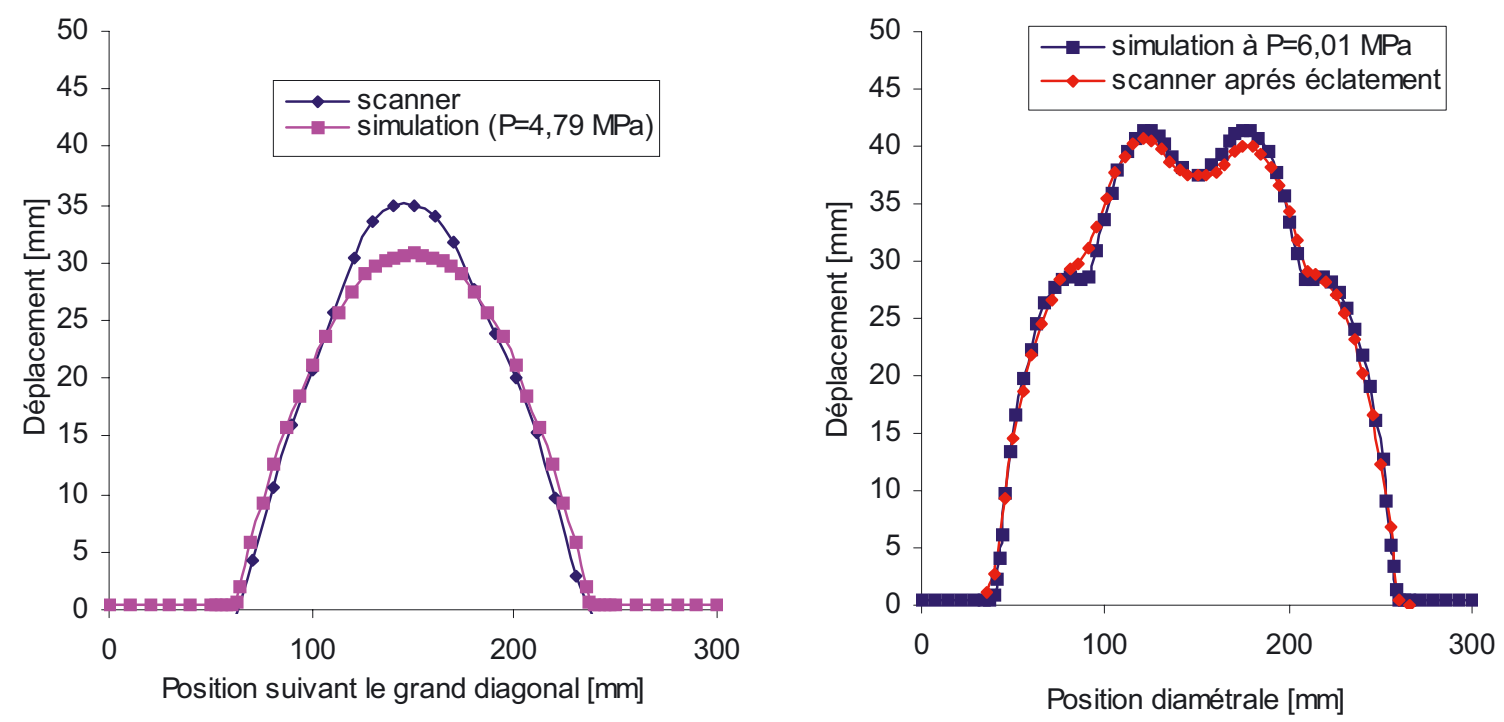

Fig. 8. Profil des déformées de la tôle, (a) essai avec serre-flan CE, (b) essai avec cavité de matrice CM3.

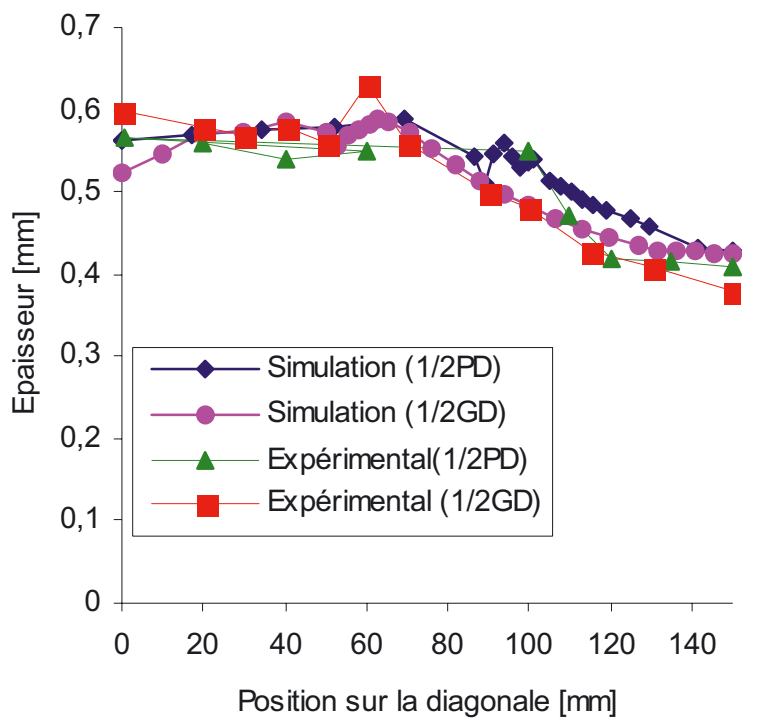

Fig. 9. Répartition des épaisseurs suivant le demi petit axe et le demi grand axe du serre-flan flan elliptique.

\subsubsection{Résultats qualitatifs}

Dans cette partie nous nous sommes intéressés à la confrontation entre les observations expérimentales des régions, où les ruptures se sont produites, et les prédictions numériques des zones concernées par l'instabilité plastique et l'endommagement. Les figures 10-14 présentent à ce titre, les principaux résultats et simulations de l'ensemble des applications traitées dans cette étude.

Les simulations des essais d'expansion libre (Figs. 10 et 11) semblent résoudre le problème de localisation des régions les plus exposées à un endommagement ductile. Les profils des contraintes équivalentes de Von Mises relevés à différents niveaux de pression permettent à la fois de localiser et d'évaluer l'étendue de la zone endommagée, celle-ci s'élève à environ $48 \mathrm{~mm}$ pour le serre-flan (CC) et à $42 \mathrm{~mm}$ suivant le grand axe pour le serre-flan (CE). Comme la simulation est effectuée à charge imposée, les effets conjugués de l'amincissement au pôle et de l'augmentation de la pression ont rendu difficile la réalisation des conditions d'équilibre local. Ceci s'est traduit numériquement par des problèmes de convergence de ce fait l'endommagement au pôle a été marqué par une chute relativement modérée, d'environ 4,5\%, de la contrainte d'écoulement.

Contrairement à l'expansion libre, les simulations des essais avec cavité de matrice (Figs. 12-14) montrent que la contrainte équivalente de Von Mises atteint des valeurs critiques élevées, pour subir ensuite une importante baisse, dans les zones endommagées. Cette baisse est évaluée pour les trois cavités de matrice CM1, CM2 et CM3 respectivement à $29 \%, 14 \%$ et à $36 \%$. En effet durant le processus de calcul, le contact de la tôle avec la matrice transforme le problème de l'équilibre local à pression imposée en un problème d'équilibre à déplacement imposé par la matrice, alors qu'une partie de la charge supportée par le flan serait transmise à la cavité. Les résultats ainsi obtenus montrent l'existence d'une corrélation entre les prédictions numériques des zones les plus exposées à l'endommagement et les zones de rupture observées expérimentalement. Les écarts de localisation entre la prédiction numérique et le résultat expérimental peuvent être imputés à la taille et au type des éléments-finis utilisés dans le modèle numérique.

Les comparaisons entre les prédictions numériques des zones endommagées et les observations expérimentales des zones de rupture nous ont conduits aux constats suivants :

1. Les calculs montrent qu'à pression croissante, les régions en expansion avec cavité de matrice sont marquées par une montée de la contrainte équivalente de Von Mises suivie d'une chute brusque, une constatation observée expérimentalement. À ce titre, les 


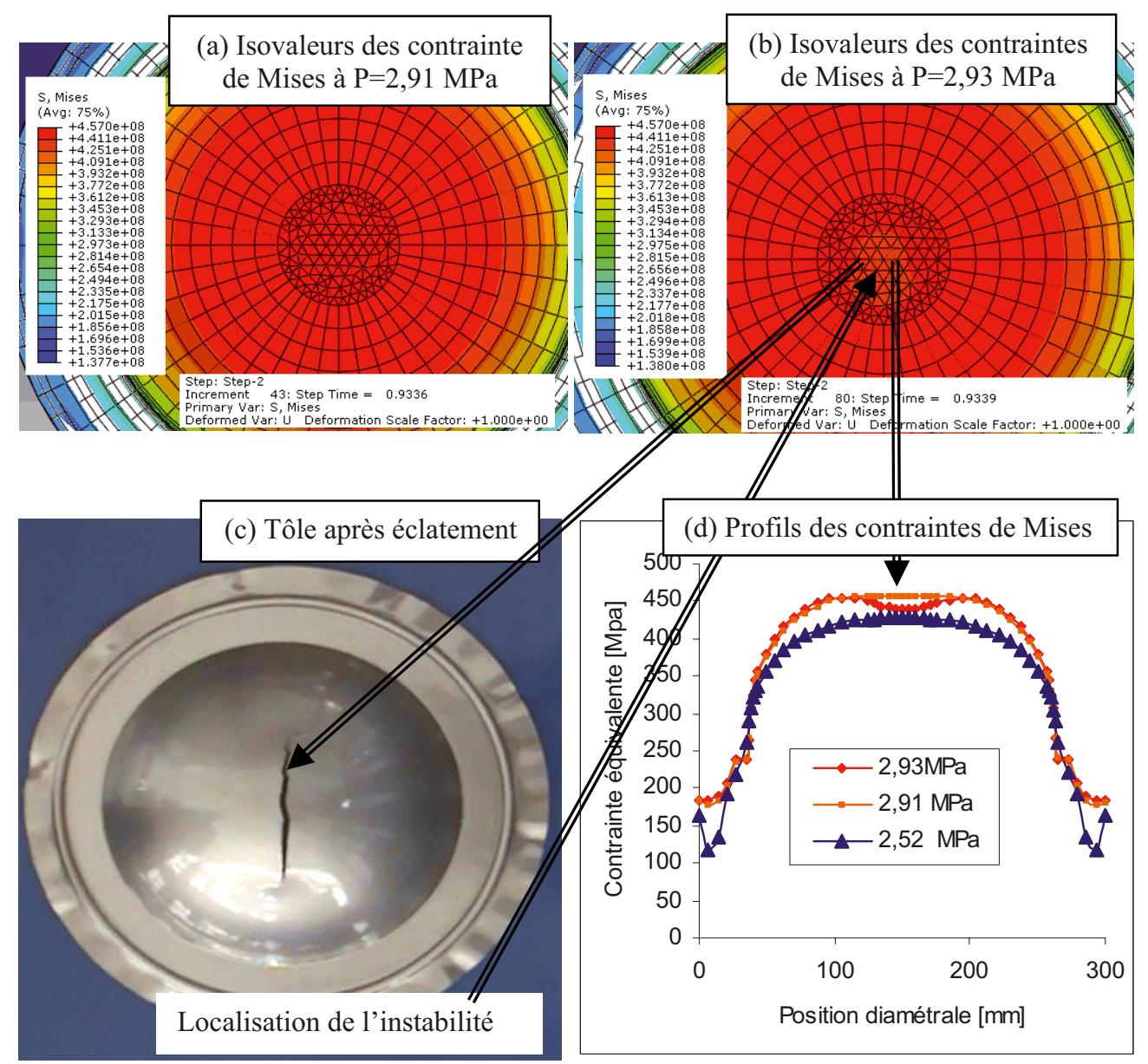

Fig. 10. Expansion libre avec serre-flan CC.

résultats relatifs à la cavité $\mathbf{C M 1}$ montrent que les instabilités ont concerné la zone centrale de la tôle, limitée par un contour circulaire de rayon de $72 \mathrm{~mm}$. La baisse la plus importante en contrainte est située dans la région délimitée par deux contours de rayons respectifs 51 et $64 \mathrm{~mm}$ et la rupture s'est produite au niveau du raccordement de la surface plane avec la surface sphérique située sur un cercle de rayon de $60 \mathrm{~mm}$. Avec la cavité CM2, la plus forte baisse de contrainte se situe entre deux contours de rayons respectifs 10 et $19 \mathrm{~mm}$, la rupture observée est située à une distance de $17 \mathrm{~mm}$ de l'axe de révolution de la tôle. Enfin pour la cavité CM3, les calculs montrent que la zone endommagée se trouve dans une région délimitée par deux contours de rayons respectifs 54 et $73 \mathrm{~mm}$, la rupture s'est produite au niveau du raccordement de la surface plane avec la surface sphérique.

2. Les prévisions numériques, avec les trois cavités de matrice prises dans l'ordre, montrent que les pressions caractérisant le début des instabilités sont respectivement de l'ordre de 4,90 $\mathrm{MPa}, 2,85 \mathrm{MPa}$ et 5,1 $\mathrm{MPa}$. Pour les applications avec les cavités CM1 et CM3, les régions, où ces débuts d'instabilité ont pu être constatés, ne correspondaient pas aux zones où se sont produites les ruptures.

\subsection{Analyse et discussion des résultats}

L'exploitation des essais expérimentaux de traction orientée et des simulations numériques a permis d'identifier le comportement élastoplastique anisotrope endommageable d'une tôle destinée à la mise en forme. Par ailleurs, la validité du modèle identifié a pu être prouvée à travers la simulation numérique d'un ensemble d'applications d'hydroformage et la comparaison des prédictions numériques aux résultats expérimentaux tels que le déplacement au pôle, la déformée de la tôle et la distribution des épaisseurs. Outre l'intérêt qu'offre le modèle identifié pour déceler les zones à risques, moyennant les simulations numériques, son exploitation peut être orientée vers l'étude de la faisabilité des formes de pièces les plus complexes en relation avec les singularités géométriques des matrices et les niveaux des chargements à mettre en ouvre. L'élaboration d'un modèle de comportement précis contribue à une analyse fiable de la faisabilité des formes désirées.

Toutefois, produire des pièces complexes, par hydroformage, qui soient résistantes et légères nécessite un contrôle rigoureux dans le temps de la pression de formage. Ceci est en effet possible grâce aux estimations des pressions annonçant, in situ, les premières instabilités 

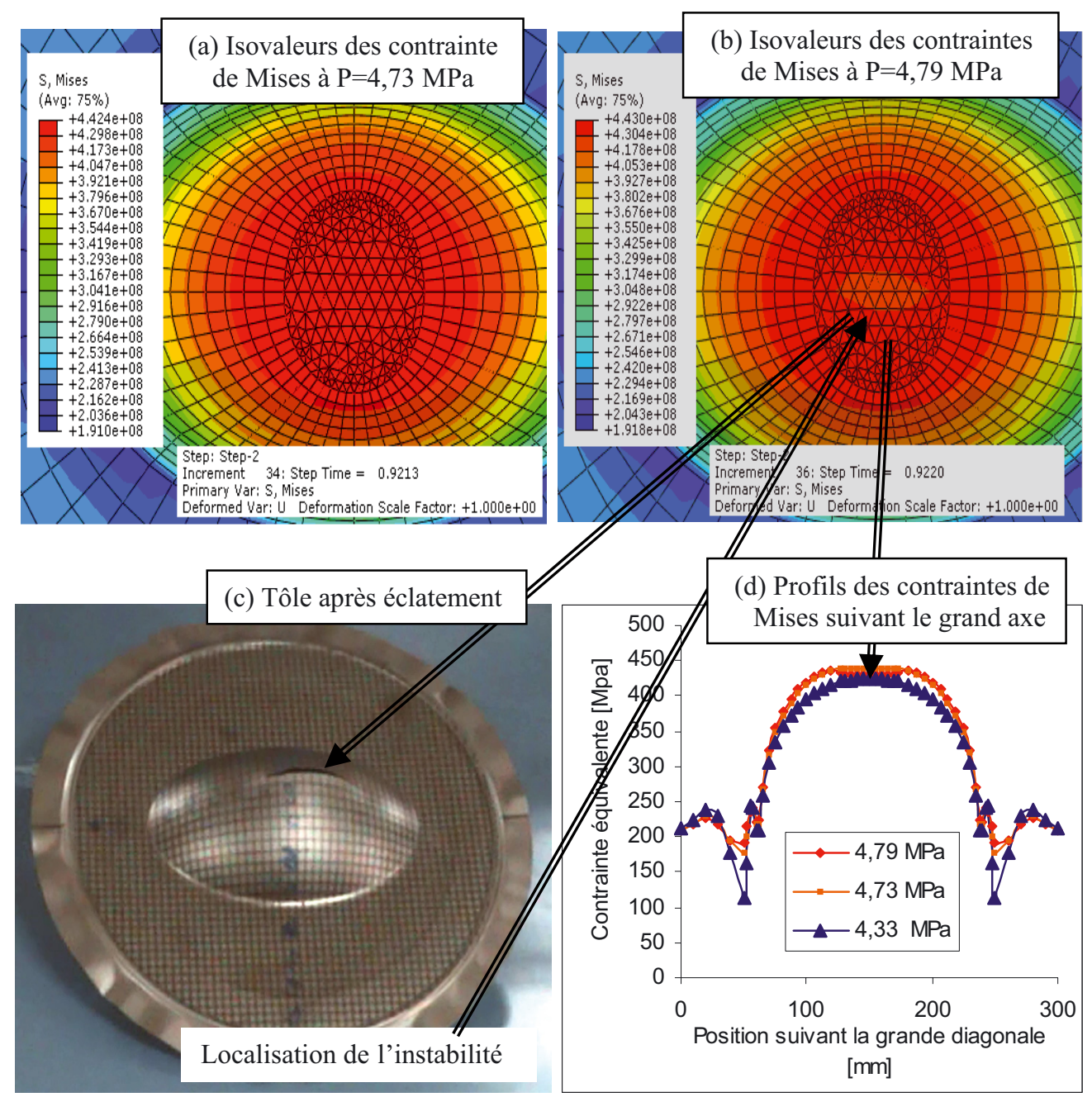

Fig. 11. Expansion libre avec serre-flan CE.

$\left(P_{\mathrm{i}}\right)$. Pour les applications développées, il y a lieu de définir aussi une pression critique $\left(P_{\mathrm{c}}\right)$, celle-ci correspond au maximum de la contrainte de Von Mises dans les régions où s'est produite la rupture. La procédure adoptée, pour déterminer ces deux pressions $\left(P_{\mathrm{i}}\right.$ et $\left.P_{\mathrm{c}}\right)$, consiste à suivre l'évolution de la contrainte équivalente en deux points de la tôle dont le premier est situé dans la région où les premières instabilités ont pu être observées et le second est situé dans la région critique localisée à la fois par expérience et par simulation. La figure 15a permet d'évaluer l'influence de la forme de la cavité sur le niveau pression critique. La figure $15 \mathrm{~b}$ montre, pour le cas de la cavité de matrice CM1, l'évolution de la contrainte de Von Mises au pôle de la plaque et en un point situé sur un cercle de rayon de $63 \mathrm{~mm}$. Le tableau 5 récapitule, pour l'ensemble des applications traitées les valeurs retenues de ces pressions. Les résultats de la prédiction numérique montrent que les pressions critiques, comparées à celles annonçant le début des instabilités sont, pour l'expansion libre, du même ordre de grandeur que la pression expérimentale d'éclatement. Cependant, les applications avec cavités de matrice mettent en évidence des écarts importants pouvant atteindre $30 \%$ entre pression critique et pression d'éclatement. En outre, on remarque que les zones concernées par les premières instabilités n'ont pas été nécessairement des zones de rupture. Ceci montre que l'évolution du dommage peut être bloquée pendant le processus de formage de la tôle surtout dans les régions de contact concernées par une atténuation des contraintes d'expansion. Le dommage se propage ainsi vers les zones en expansion qui subissent directement l'effet croissant de la pression hydraulique.

\section{Conclusion}

Les investigations menées dans cette étude montrent l'intérêt d'entreprendre la caractérisation des aptitudes de formage des tôles sur la base d'essais de traction orientée. Il semble que l'exploitation des diagrammes forces/déplacements relatifs aux essais de traction pour le calage des modèles d'écrouissage couplé à l'endommagement est susceptible de fournir des solutions satisfaisantes pour l'identification du comportement des tôles. 


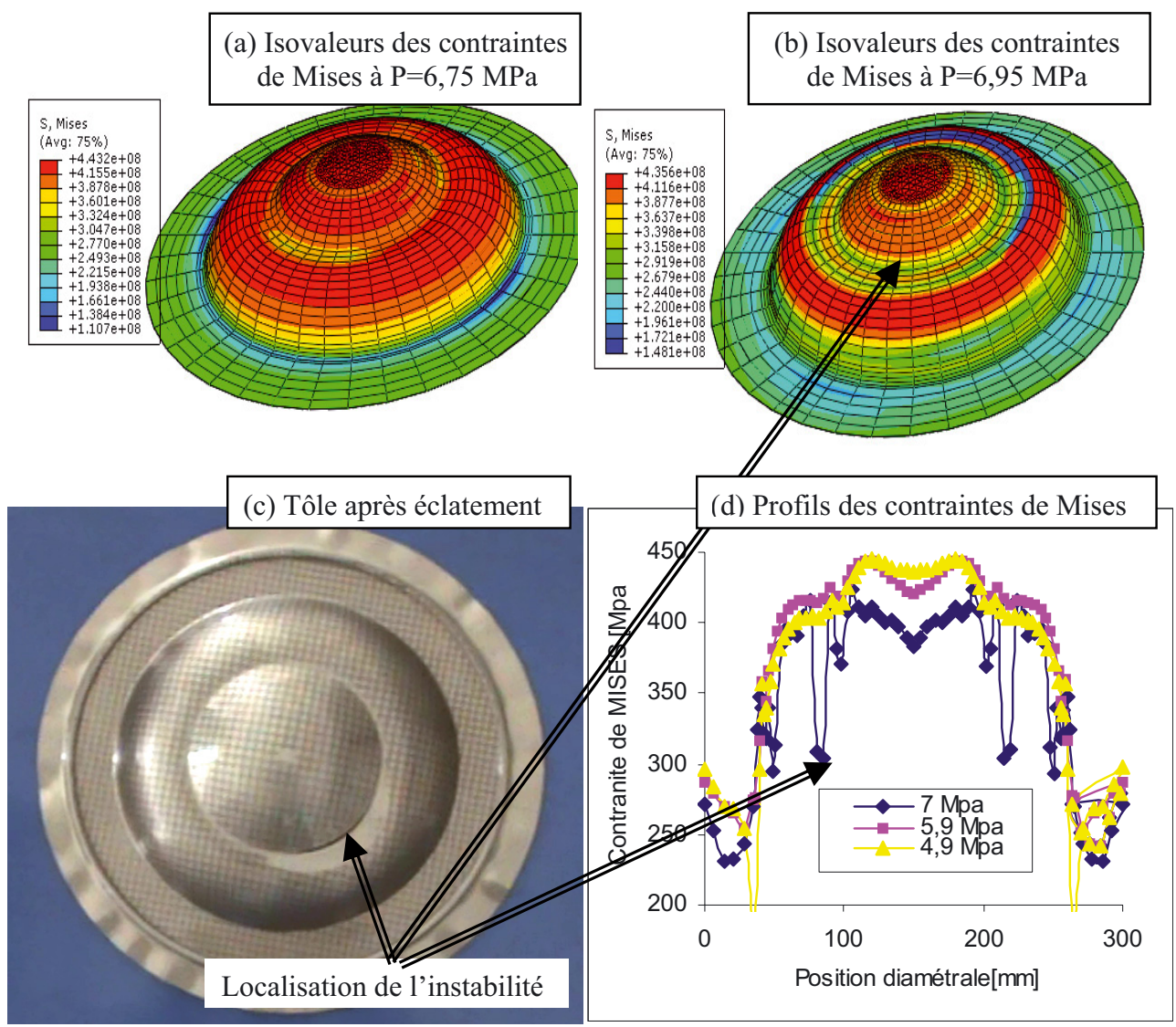

Fig. 12. Expansion dans une cavité de matrice type CM1.

Tableau 4. Comparaison expérience/simulation des essais de traction orientée.

\begin{tabular}{|c|c|c|c|c|}
\hline & & Expérience & Simulation & Écart relatif [\%] \\
\hline & $\begin{array}{c}\text { Tension maximale }[\mathrm{N}] \\
\text { (16,7 mm d'allongement) }\end{array}$ & 2194 & 2191 & 0,14 \\
\hline $0^{\circ}$ & $\begin{array}{l}\text { Allongement à la rupture [mm] } \\
(1470 \mathrm{~N} \text { de tension })\end{array}$ & 25,18 & 26,50 & 5,24 \\
\hline & $\begin{array}{c}\text { Tension maximale }[\mathrm{N}] \\
(20,1 \mathrm{~mm} \text { d'allongement }) \\
\end{array}$ & 2345 & 2421 & 3,20 \\
\hline $45^{\circ}$ & $\begin{array}{l}\text { Allongement à la rupture [mm] } \\
(1859 \mathrm{~N} \text { de tension })\end{array}$ & 26,4 & 23,5 & 9,60 \\
\hline \multirow[b]{2}{*}{$90^{\circ}$} & $\begin{array}{c}\text { Tension maximale }[\mathrm{N}] \\
(19,8 \mathrm{~mm} \text { d'allongement) }\end{array}$ & 2089 & 2097 & 2,4 \\
\hline & $\begin{array}{l}\text { Allongement à la rupture [mm] } \\
\text { (1190 N de tension) }\end{array}$ & 24,5 & 28,1 & 14,7 \\
\hline
\end{tabular}

Tableau 5. Niveaux des pressions dans les différents cas d'application.

\begin{tabular}{cccc}
\hline $\begin{array}{c}\text { Type } \\
\text { d'application }\end{array}$ & $\begin{array}{c}\text { Pression au début des } \\
\text { instabilités }[\mathrm{MPa}]\end{array}$ & $\begin{array}{c}\text { Pression critique } \\
{[\mathrm{MPa}]}\end{array}$ & $\begin{array}{c}\text { Pression expérimentale à } \\
\text { l'éclatement [MPa] }\end{array}$ \\
\hline CC & 2,93 & 2,92 & 3,2 \\
CE & 4,79 & 4,8 & 4,9 \\
CM1 & 4,90 & 6,74 & 5,2 \\
CM2 & 2,85 & 2,85 & 3,0 \\
CM3 & 5,10 & 6,86 & 5,3 \\
\hline
\end{tabular}



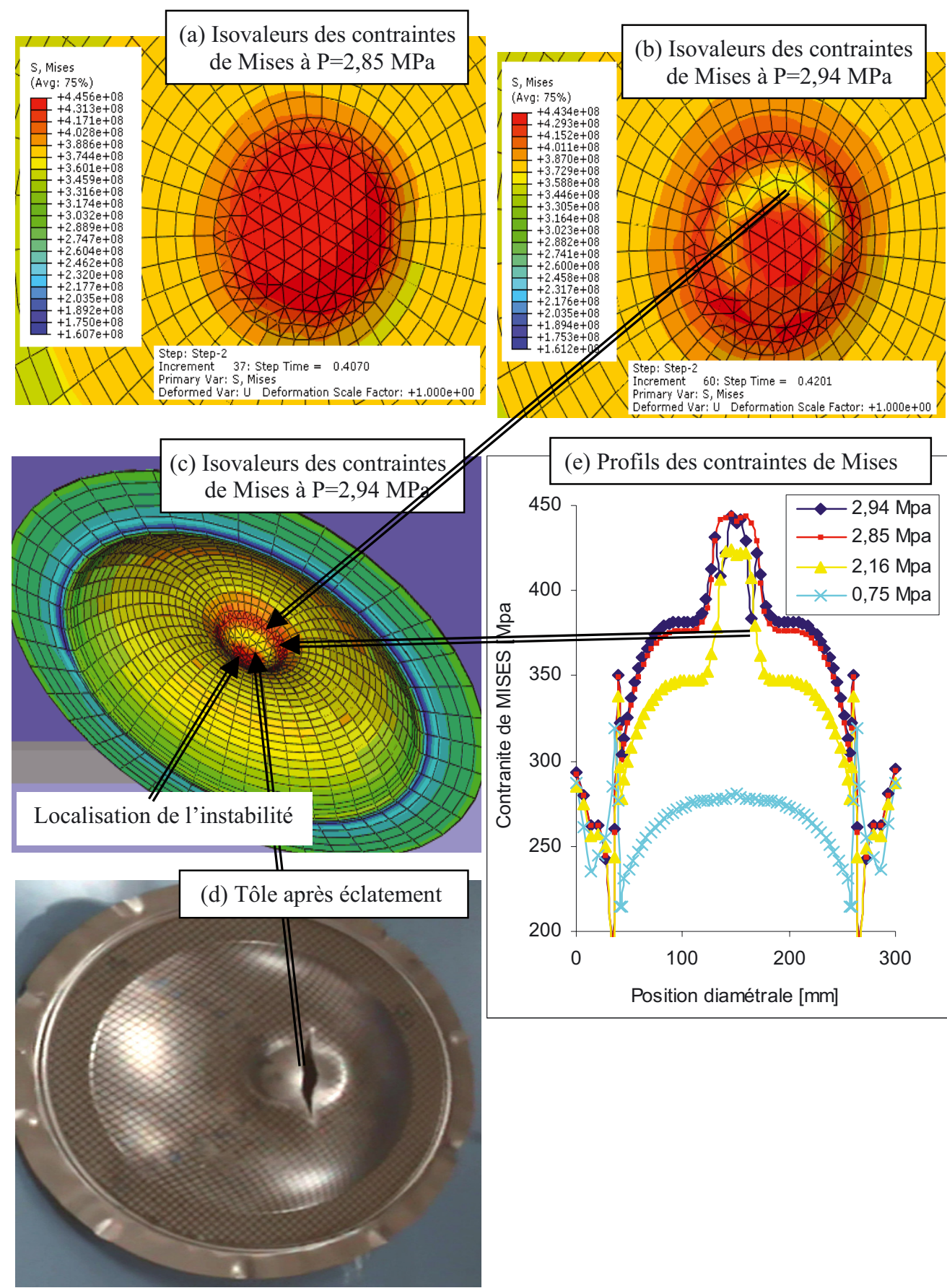

(e) Profils des contraintes de Mises

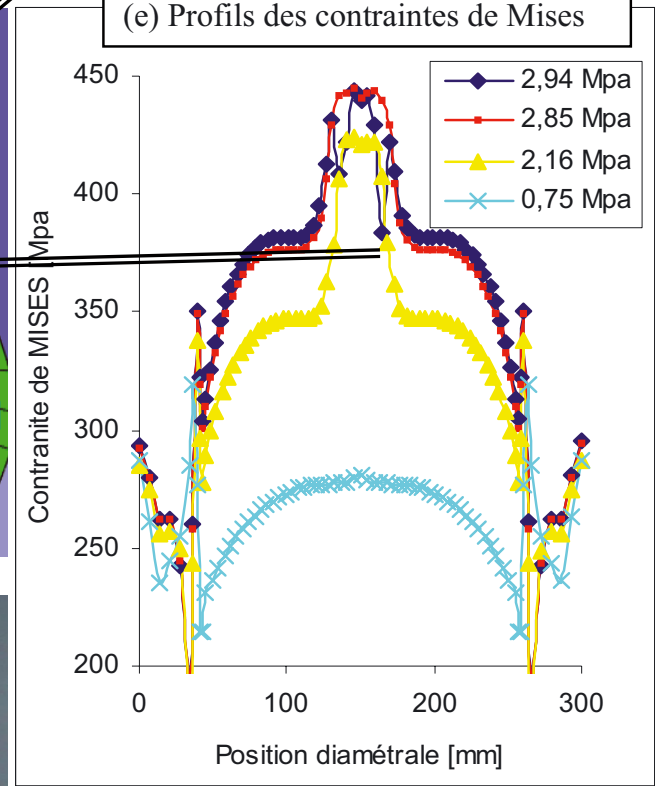

Fig. 13. Expansion dans une cavité de matrice type CM2.

Nous avons montré que la prise en compte de l'anisotropie initiale de la tôle et l'utilisation, dans les simulations numériques, d'un modèle d'écrouissage couplé à l'endommagement ductile permet la localisation des zones d'instabilité et l'évaluation de l'étendue du dommage. Moyennant un modèle d'écrouissage couplé à l'endommagement fidèle et précis, la simulation numérique pourra contribuer à une amélioration de la faisabilité des procédés de mise en forme des structures minces. Cette amélioration concernera l'analyse de la faisabilité des géométries complexes en relation avec les singularités géométriques des cavités des matrices, des aptitudes de formage des tôles et du chemin de chargement durant le processus de formage.

Les orientations futures de cette étude porteront sur l'utilisation des outils de maillage et de remaillage adaptatif pour prédire les régions exposées à la rupture et l'évaluation expérimentale et numérique de la distribution du dommage ainsi que la détermination de la force thermodynamique associée à l'endommagement [30]. À 


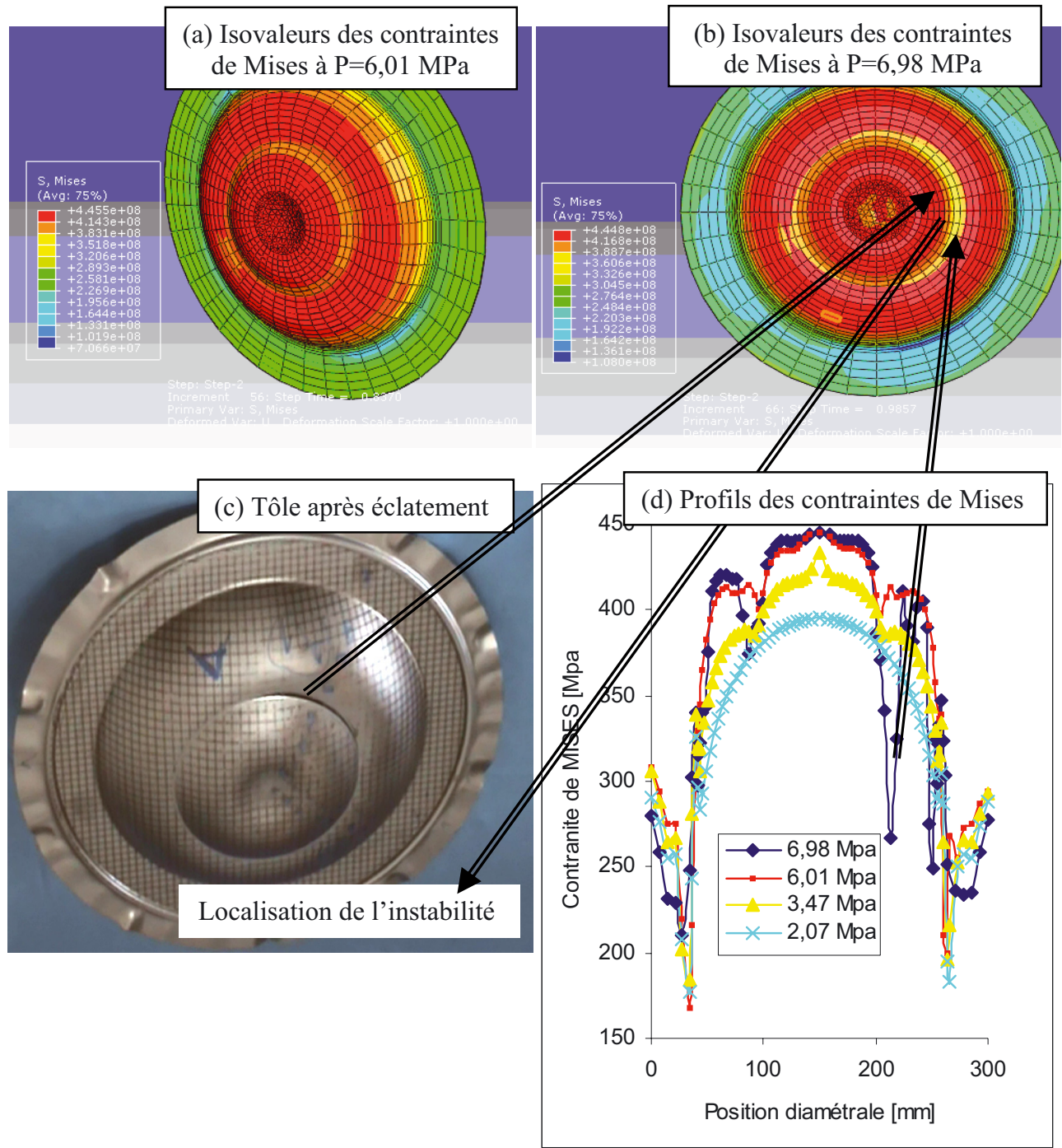

Fig. 14. Expansion dans une cavité de matrice type CM3.
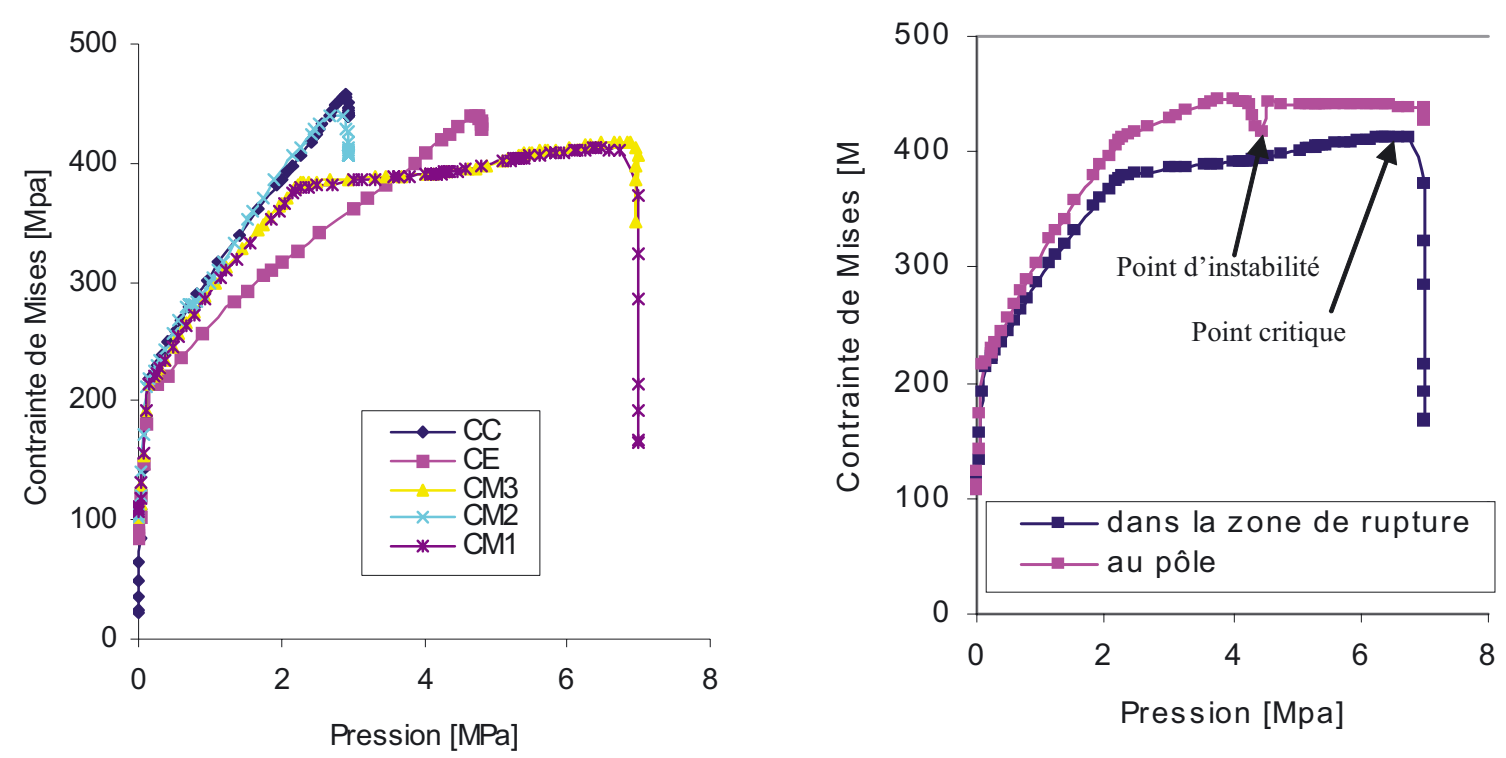

Fig. 15. Contrainte équivalente en fonction de la pression, (a) dans les zones de rupture; (b) en deux points du flan CM1. 
cet effet, la nano-indentation pourra se présenter en tant que technique expérimentale de mesure et d'exploration de l'endommagement [25].

\section{Références}

[1] E.D. Mielnieck, Metal working science and engineering, Mc Graw-Hill, Inc, 1991

[2] K. Saanouni, J.-L. Chaboche, Computational damage mechanics: application to metal forming, Chap. 7, Vol. 3, Numerical and computational methods, I. Miline, R.-O. Ritchie, B. Karihalo (Eds.), ISBN 0-08-043749-4, Elsevier Oxford 2003, pp. 3211-376

[3] A.L. Gurson, Continuum theory of ductile rupture by void nucleation and growth: Part I, Yield criteria and flow rules for porous ductile media, J. Eng. Mat. Tech. 99 (1997)

[4] G. Cailletaud, Une approche micromécanique phénoménologique du comportement inélastique des métaux, Thèse de Docteur d'Etat, Université Paris VI, 1987

[5] A. Zaoui, Comportement global des polycristaux, Passage du polycristal au monocristal, Phys. et mécanique de la mise en forme des métaux, Presse du CNRS IRSID, 337, 1990

[6] D. François, A. Pineau A. Zaoui, Comportement Mécanique des Matériaux. Endommagement, Mécanique de la Rupture, Mécanique de contact, Vol. 2 Hermes, 1993

[7] J. Lemaitre, A continuum damage mechanics model for ductile fracture, J. Eng. Mat. Tech. 107 (1985) 83-89

[8] P. Germain, Q.-S. Nguyen, P. Suquet, A Continuum thermodynamics, J. Eng. Mech. 50 (1983) 1010-1020

[9] J.-R. Rice, D-M. Tracy, On the enlargement of voids in triaxial stress field J. Mech. Phys. 17 (1969) 201-217

[10] G. Pijaudier-Cabot, Z.P. Bazant, Nonlocal damage theory. J. Engrg. Mech. 113 (1987) 1512-1533

[11] R. Becker, A. Needleman, O. Richmond, G. Tvergaard, Void growth and failure in notched bars, J. Mech. Phys. 36 (1988) 317-351

[12] V. Tvergaard, Material failure by void growth to coalescence, J. Adv. Appl. Mech 27 (1990) 83-151

[13] J.-L. Chaboche, Continuum damage mechanics, Part I \& II, J. Applied Mechanics 55 (1988) 59-72

[14] L.M. Kachanov, Time of the rupture process under creep conditions, Z.v, Akad, Nauk. SSR 8 (1958) 26

[15] I.M. Pereiraa, G. Rubimb, O. Acselradc, P.R. Cetlin, Comparison of the experimental and the numerically predicted mechanical damage in the sheet forming of steel, J. Mater. Proc. Tech. 203 (2008) 13-18
[16] J.C. Gelin, Modelling of damage in metal forming processes J. Mat. Processing Tech. 80-81 (1998) 24-32

[17] P. Teixeira, A.-D. Santos, F.M. Andrade Pires, J.-M.A.C. Santos, Finite element prediction of ductile fracture in sheet metal forming processes, J. Mat. Processing Tech. 177 (2006) 278-281

[18] A. Cherouat, K. Saanouni, Y. Hammi, Numerical improvement of thin tubes hydroforming with respect to ductile damage, I. J. Mech. Sci. 44 (2002) 2427-2446

[19] R. Hill, A theory of yielding and plastic flow of anisotropic metals, Royal Soc. London Proc. (1948) 281

[20] N. Siva, P. Varma, R. Narasimhan, A.A. Luo, A.K. Sachdev, An analysis of localized necking in aluminium alloy tubes during hydroforming using a continuum damage model, I. J. Mech. Sci. 49 (2007) 200-209

[21] F.M. Andrade Pires, E.A. de Souza Neto, D.R.J. Owen, On the finite element prediction of damage growth and fracture initiation in finitely deforming ductile materials, Computer Methods in Applied Mechanics and Engineering 193 (2004) 5223-5256

[22] A. Imad, On the ductile fracture based on the continuum damage in a steel case, Mécanique \& Industries 3 (2002) $45-50$

[23] ABAQUS, Theory Manual, Version 6.7, Hibbit, Karson \& Sorensen, 2008

[24] M. Khelifa, M. Oudjene, Numerical damage prediction in deep-drawing of sheet metals, J. Mater. Processing Tech. (2008) 1-76

[25] M. Ayadi, H. Cherouat, M.A. Rezgui, N. Mezghani, Experimental and numerical studies of welded tube formability. J. Mat. Sci. Forum 614 (2009) 129-134

[26] N. Mezghani, H. Salhi, M. Ayadi, A. Cherouat, Experimental and numerical simulation of hydroforming process, Int. Rev. Mech. Eng. 2N6 (2008) 839-844

[27] MATLAB Users Manual, The Mathworks Inc., 2007

[28] Simon Ho, Yung-Li Lee, Hong-Tae Kang, Cheng J. Wang, Optimization of a crankshaft rolling process for durability, Int. J. Fatigue 31 (2009) 799-808

[29] Jiromaru Tsujino, Kazuaki Hidai, Atsushi Hasegawa, Ryoichi Kanai, Hisanori Matsuura, Kaoru Matsushima, Tetsugi Ueoka, Ultrasonic butt welding of aluminium, aluminium alloy and stainless steel plate specimens Ultrasonics 40 (2002) 371-374

[30] L. Giraud-Moreau, H. Borouchaki, A. Cherouat, A Remeshing Procedure for Numerical Simulation of Forming Processes in Three Dimensions, New York, Springer ISBN 3-540-34957-X, 2006 127-143 